\title{
Transatlantica
}

Revue d'études américaines. American Studies Journal

\section{La bande dessinée, nouveau continent des études américaines? Avant-propos}

Jean-Paul Gabilliet

\section{(2) OpenEdition}

1 Journals

\section{Édition électronique}

URL : https://journals.openedition.org/transatlantica/4937

DOI : $10.4000 /$ transatlantica.4937

ISSN : $1765-2766$

Éditeur

Association française d'Etudes Américaines (AFEA)

\section{Référence électronique}

Jean-Paul Gabilliet, "La bande dessinée, nouveau continent des études américaines ? Avant-propos », Transatlantica [En ligne], 1 | 2010, mis en ligne le 29 septembre 2010, consulté le 10 février 2023. URL: http://journals.openedition.org/transatlantica/4937 ; DOI : https://doi.org/10.4000/transatlantica. 4937

Ce document a été généré automatiquement le 10 février 2023.

Creative Commons - Attribution - Pas d'Utilisation Commerciale - Pas de Modification 4.0 International - CC BY-NC-ND 4.0

https://creativecommons.org/licenses/by-nc-nd/4.0/ 


\title{
La bande dessinée, nouveau continent des études américaines? Avant-propos
}

\author{
Jean-Paul Gabilliet
}

1 Lors d'une table ronde qui s'est tenue en juillet 2009 pendant la San Diego Comic-Con (équivalent américain du Festival international de la bande dessinée d'Angoulême), Henry Jenkins, professeur de sciences de la communication à University of Southern California, a salué le processus actuel de construction des Comic Studies comme domaine de recherche et d'enseignement inter-disciplinaire et souligné la pluralité des devenirs qui pouvaient s'ouvrir devant lui (http://henryjenkins.org/2009/07/ the_first_thing_about_san.html). Etablissant des comparaisons avec des segments de la culture populaire ayant déjà accédé à l'Université, il a rappelé qu'aux Etats-Unis, les études cinématographiques ont contribué à élargir les connaissances et les goûts du public en matière cinéphilique de même que les études sur les jeux vidéo (Game Studies) ont contribué à alimenter le débat sur la liberté d'expression. Mais, en parallèle à ces évolutions "positives", Jenkins a souligné que l'étude de la science-fiction avait essentiellement pris racine dans des départements de lettres où n'étaient étudiées que les œuvres les plus « littéraires », aux dépens du corpus pourtant majoritaire des textes issus de l'édition populaire (la pulp fiction au sens le plus fort de cette expression), certes sans mérites "littéraires" (tout au moins du point de vue l'ethnocentrisme lettré) mais d'un intérêt considérable en matière d'histoire culturelle et de la communication. D'où une mise en garde : l'étude de la bande dessinée doit par tous les moyens éviter de s'échouer sur un écueil avant-gardiste. Aussi intellectuellement respectable soit-il, un tel processus risquerait fort d'aboutir à la légitimation de certaines bandes dessinées par des critiques littéraires ou critiques d'art, mais toujours à un niveau subalterne par rapport au socle de référence de la littérature et des beauxarts.

Quoiqu'on ait tendance à l'oublier, cela fait maintenant presque un demi-siècle que les voix les plus diverses - en France, en Amérique du nord et maintes autres contrées - 
annoncent que la bande dessinée est "enfin" devenue digne de l'attention des universitaires. Il s'agit néanmoins d'un domaine où c'est la preuve par l'exemple, et non par l'institution, qui garde force de loi : s'il ne viendra à l'idée de personne (on peut l'espérer...) de contester la légitimité intellectuelle et scientifique d'une livraison de Transatlantica consacrée à l'étude de la bande dessinée dans le domaine nord-américain, ce moyen d'expression n'a pas encore atteint le stade qui en fasse un secteur universitaire à part entière, à l'image des prestigieux prédécesseurs à composante visuelle qu'ont été la photographie et le cinéma. Ceux-ci constituent le socle des visual arts sur lequel sont venus s'agréger toute sorte de médias visuels (la télévision, la vidéo, les jeux vidéo, etc.) mais la bande dessinée reste - curieusement ? - marginale dans cette ensemble. Pour quelle raison ? Peut-être la BD, de manière contre-intuitive, n'estelle pas aussi « visuelle » que ça. Elle est aussi très narrative, y compris quand elle est " muette » - après tout, le cinéma était déjà essentiellement narratif, même avant de devenir parlant. Cette hybridité structurelle, qui est en soi un mauvais point dans les hiérarchies culturelles occidentales (mais pas au Japon, par exemple) aboutit à ce que le média considéré se trouve simultanément à intéresser pour ses aspects formels les spécialistes de l'écrit et ceux de l'image. Ajoutons à cela le regard porté sur l'histoire de ce moyen d'expression par historiens et spécialistes d'"info-com" et on aboutit à un domaine de savoir au moins partageable entre quatre approches scientifiques, auxquelles peuvent s'adjoindre sans grande difficulté des perspectives anthropologiques, philosophiques, juridiques, etc.

Rien de bien neuf ici. La bande dessinée n'est pas - loin de là - le seul moyen d'expression qui soit une espèce narrative et en même temps se déploie au sein d'un secteur identifiable de l'industrie culturelle (aussi tentaculaire puisse-t-il paraitre aux non-initiés). Mais elle continue à rencontrer des résistances qui se manifestent de diverses manières. Par exemple : comment s'intitule l'étude universitaire de la bande dessinée? Etudes bédéistiques? Comicologie? Le français peine à trouver une terminologie qui ramasse en un seul mot ou une formule courte tout ce domaine de savoir. La langue anglaise, plus adaptable que la nôtre, a formé sans scrupule quant à de possibles ambiguïtés le composé Comic Studies, parfois relevé en Comic Art Studies.

4 Les Comic Studies existent-elles en Amérique du nord ? Oui... en quelque sorte. Disons qu'un certain nombre de signes manifestent la montée d'une dynamique universitaire autour des questions relatives à la bande dessinée: une collection spécialisée chez University Press of Mississippi fondée par M. Thomas Inge il y a une vingtaine d'années, la revue papier International Journal of Comic Art animée depuis 1999 par John A. Lent à Temple University (http://www.ijoca.com/IJOCA.htm), la revue en ligne ImageTexT, sous-titrée "Interdisciplinary Comics Studies" (http://www.english.ufl.edu/ imagetext/) depuis 2004, la liste de discussion "Comics Scholars' List" (http:// www.english.ufl.edu/comics/scholars/) - toutes deux basées à l'Université de Floride à Gainesville-, un nombre croissant de colloques dans le monde entier et de cours proposés dans des départements de littérature, de communication, d'histoire voire de philosophie, sans compter la publication à l'été 2009 du premier textbook à destination du public universitaire, The Power of Comics: History, Form and Culture de Randy Duncan et Matthew J. Smith (Continuum, 2009). A l'évidence, beaucoup de chemin a été parcouru depuis la première soutenance d'une thèse de doctorat consacrée à la bande dessinée en 1959 à NYU (Davidson). Mais... il n'existe toujours pas de département d'université consacré à la BD et celle-ci continue de constituer un choix professionnel à haut risque qui n'ouvre pas les portes des grandes universités, ni même des petites, à l'enseignant- 
chercheur qui ne peut pas faire état d'un sujet de spécialisation plus « légitime ». Il est vrai que quand des établissements mondialement renommés en arrivent à fermer des départements de lettres et/ou de SHS, le recrutement de chercheurs spécialisés en BD n'apparaît pas comme une priorité scientifique ou économique...

5 Abstraction faite du débat institutionnel, une question subsiste : si la bande dessinée peut être un objet d'étude et de savoir, comment l'étudier et comment construire le(s) savoir(s) qui en rend(ent) compte? Ce questionnement entraîne des réponses complexes qu'il est imprudent d'éluder. Pour en avoir une idée plus précise, on peut se référer à l'essai critique publié en 2004 dans le Canadian Journal of Communication où Bart Beaty mettait en exergue un certain nombre de faiblesses chroniques (ainsi que quelques points forts, heureusement) d'ouvrages "universitaires" récents sur la bande dessinée. Il n'est pas intutile de rappeler qu'en Amérique du nord en particulier, le moyen d'expression qui nous intéresse ici a connu un développement historique extrêmement nuancé. Il est passé du livre humoristique et/ou pour enfants au milieu du XIXe siècle à la presse satirique pendant l'Age Doré. Il est devenu un divertissement familial dans la presse quotidienne au tournant du XXe siècle puis un des piliers de l'édition populaire à partir de la fin des années 30. Utilisé comme un des modes privilégiés d'expression de la mouvance contre-culturelle dans les années 60 et 70, il a finalement accouché à la fin du XXe siècle d'une nouvelle forme, le «roman graphique", qui a consacré la possibilité enfin donnée à des créateurs de bande dessinée de faire un travail d'auteur, à la façon des romanciers et cinéastes.

Ce panorama fort rapide permet néanmoins de mettre la doigt sur la complexité des enjeux scientifiques qui président à la construction des Comic Studies. Si le moyen d'expression fait preuve d'une certaine stabilité depuis ses débuts - encore que les critères de définition de la bande dessinée peuvent faire l'objet de débats sans fin, comme j'ai eu l'occasion d'en parler ailleurs (Gabilliet 11-17)-, les usages qui en ont été faits par éditeurs, créateurs et lecteurs (n'oublions pas les lecteurs!) depuis deux siècles sont, eux, fort divers. D'où la nécessité de permettre la coexistence d'approches formelles et historiques : un truisme étant souvent la voie la plus sûre vers la sagesse, il n'est pas inutile de rappeler que, dans son hétérogénéité spatiale et temporelle, la bande dessinée est produite par des individus, des cultures, des époques, des contextes et des pratiques. C'est donc un regard multi-directionnel qui est présenté dans cette livraison de Transatlantica qui propose successivement un état des lieux documentaire réalisé par un archiviste (Rhode), deux études de cas consacrées l'une à une bande dessinée d'auteur (Merino), l'autre à des récits de super-héros (Labarre), un entretien de Bart Beaty avec deux pédagogues (Abel et Madden), une réflexion sur la manière de penser la sérialité en rapport avec la bande dessinée (Baetens), deux analyses du champ en devenir des Comic Studies (Hatfield, Fischer) et un essai bibliographique (Gabilliet). La variété de perspectives stimulantes sur l'étude de la bande dessinée, que celle-ci soit nord-américaine, européenne, asiatique ou autre, ici proposées donnera au lecteur un aperçu du champ de réflexion qui se déploie depuis quelques années autour de cet objet pourtant déjà ancien en termes purement historiques. 


\section{BIBLIOGRAPHIE}

BEATY, Bart, "Review Essay : Assessing Contemporary Comics Scholarship", Canadian Journal of Communication 29 (2004), 403-09. Téléchargeable à l'adresse http://www.cjc-online.ca/index.php/ journal/article/download/1485/1604.

DAVIDSON, Sol M., "Culture and the Comic Strips”, diss. phil., New York University, 1959.

GABILLIET, Jean-Paul, Des Comics et des hommes : histoire culturelle des comic books aux Etats-Unis, Nantes, Ed. du Temps, 2005.

JENKINS, Henry, "My Wild and Wonderful Comic-Con Experiences", Confessions of an Aca-Fan website,http://henryjenkins.org/2009/07/the_first_thing_about_san.html, 19 août 2009.

\section{AUTEUR}

JEAN-PAUL GABILLIET

Université Bordeaux III 